\title{
Meta-Analysis Comparing Renal Outcomes after Transcatheter versus Surgical Aortic Valve Replacement
}

\author{
Kuldeep Shah $\mathbb{D}$, ${ }^{1}$ Zakeih Chaker, ${ }^{2}$ Tatiana Busu, ${ }^{2}$ Rishita Shah $\mathbb{D},{ }^{1}$ Mohammed Osman $(\mathbb{D}$, \\ Fahad Alqahtani, ${ }^{1}$ and Mohamad Alkhouli ${ }^{1}{ }^{1}$ \\ ${ }^{1}$ Division of Cardiology, West Virginia School of Medicine, Morgantown, WV, USA \\ ${ }^{2}$ Department of Medicine, West Virginia School of Medicine, Morgantown, WV, USA \\ Correspondence should be addressed to Mohamad Alkhouli; mohamad.alkhouli@wvumedicine.org
}

Received 19 November 2018; Accepted 7 April 2019; Published 24 April 2019

Academic Editor: Vasileios Panoulas

Copyright (C) 2019 Kuldeep Shah et al. This is an open access article distributed under the Creative Commons Attribution License, which permits unrestricted use, distribution, and reproduction in any medium, provided the original work is properly cited.

Background. Acute kidney injury (AKI) is a common complication of aortic valve replacement. However, comparative on the incidence of (AKI) following transcatheter (TAVR) versus surgical valve replacement (SAVR) is sparse. Methods. We performed a meta-analysis of the randomized controlled trials (RCT) and propensity-matched observational studies comparing (A) incidence of AKI and (B) incidence of dialysis-requiring AKI at 30 days after TAVR and SAVR. Results. Twenty-six studies (20 propensitymatched studies; 6 RCTs) including 19,954 patients were analyzed. The incidence of AKI was lower after TAVR than after SAVR (7.1\% vs. $12.1 \%$, OR $0.52 ; 95 \% \mathrm{CI}, 0.39-0.68 ; \mathrm{p}<0.001, \mathrm{I}^{2}=57 \%$ ), but the incidence of dialysis-requiring AKI was similar $(2.8 \%$ vs. $4.1 \%$, OR $\left.0.78 ; 95 \% \mathrm{CI}, 0.49-1.25 ; \mathrm{p}=0.31, \mathrm{I}^{2}=70 \%\right)$. Similar results were observed in a sensitivity analysis including RCTs only for both AKI ([5 RCTs; 5,418 patients], $2.0 \%$ vs. 5.0\%, OR 0.39; 95\%CI, 0.28-0.53; $\mathrm{p}<0.001, \mathrm{I}^{2}=0 \%$ ), and dialysis-requiring AKI ([2 RCTs; 769 patients]; $2.9 \%$ vs. $2.6 \%$, OR 1.1; $\left.95 \% \mathrm{CI}, 0.47-2.58 ; \mathrm{p}=0.83, \mathrm{I}^{2}=0 \%\right)$. However, in studies including low-intermediate risk patients only, TAVR was associated with lower incidence of AKI ([10 studies; 6,510 patients], 7.6\% vs. 12.4\%, OR 0.55, 95\%CI 0.39-0.77, p<0.001, $\mathrm{I}^{2}=57 \%$ ), and dialysis-requiring AKI, ([10 studies; 12,034 patients], $2.0 \%$ vs. $3.6 \%$, OR $0.57,95 \% \mathrm{CI} 0.38-0.85, \mathrm{p}=0.005, \mathrm{I}^{2}=23 \%$ ). Conclusions. TAVR is associated with better renal outcomes at 30 days in comparison with SAVR, especially in patients at lowintermediate surgical risk. Further studies are needed to assess the impact of AKI on long-term outcomes of patients undergoing TAVR and SAVR.

\section{Introduction}

The introduction of transcatheter aortic valve replacement (TAVR) and the continuous improvement in the outcomes of surgical aortic valve replacement (SAVR) have revolutionized the treatment of patients with severe aortic stenosis in the last decade [1, 2]. However, acute kidney injury (AKI) remains a common complication of both treatment modalities. Nonetheless, data on the incidence of AKI and dialysis-requiring AKI after TAVR vs. SAVR remain limited [3]. We performed a meta-analysis of the randomized clinical trials (RCTs) and propensity-matched (PSM) observational studies to compare renal outcomes following TAVR vs. SAVR (a) overall and (b) in subgroups of high-risk and lowintermediate risk patients.

\section{Methods}

Our review protocol was conducted in accordance with PRISMA (Preferred Reporting Items for Systematic Reviews and Meta-Analyses) reporting guidelines (Supplementary Protocol). [4] The literature search was conducted in PUBMED, MEDLINE, EMBASE, EBSCO, and Cochrane (March 2, 2018) in order to identify eligible studies using the Medical Subject Headings search terms and text word search. We also did a manual search of the reference lists of relevant studies for additional publications and when multiple publications from the same study population were found, data from the most inclusive report was used. The data was reviewed independently from full-text articles by 2 of the authors (T.B. and K.S.). Disagreements were 
TABLE 1

\begin{tabular}{lccc}
\hline Baseline Characteristics & TAVR & SAVR & p-value \\
Age (years) & (N=10,038) & $7,916)$ & 0.528 \\
Male & $79.1 \pm 5.8$ & $50.16 \%$ & 0.798 \\
Coronary artery disease & $49.17 \%$ & $52.99 \%$ & 0.606 \\
Chronic kidney disease (GFR $<60 \mathrm{~mL} / \mathrm{min})$ & $57.7 \%$ & $25.3 \%$ & 0.943 \\
Diabetes mellitus & $25.9 \%$ & $31.2 \%$ & 0.768 \\
Atrial fibrillation & $30.0 \%$ & $28.6 \%$ & 0.991 \\
Chronic obstructive pulmonary disease & $28.6 \%$ & $22.7 \%$ & 0.927 \\
Frailty & $23.0 \%$ & $27.5 \%$ & 0.957 \\
Left ventricular ejection fraction & $28.4 \%$ & $55.3 \pm 9.0$ & 0.701 \\
Pulmonary hypertension & $56.41 \pm 6.6$ & $18.7 \%$ & 0.729 \\
Peripheral vascular disease & $21.1 \%$ & $22.6 \%$ & 0.630 \\
Prior stroke or transient ischemic attack & $24.4 \%$ & $15.8 \%$ & 0.838 \\
NYHA III or IV & $16.5 \%$ & $67.73 \%$ & 0.515 \\
Prior coronary artery bypass graft & $71.4 \%$ & $31.8 \%$ & 0.445 \\
STS score & $40.9 \%$ & $6.1 \pm 2.3$ & 0.590 \\
Euro SCORE & $6.6 \pm 2.9$ & $15.0 \pm 6.3$ & 0.357 \\
\hline
\end{tabular}

resolved through consensus and arbitration by the senior author (M.A.). The following criteria were applied for study inclusion: (1) randomised controlled trials and propensitymatched observational studies comparing TAVR and SAVR; (2) being published in peer-reviewed journals; (3) followup of at least 30 days; and (4) reporting AKI or acute renal failure and/or new requirement for renal replacement therapy (dialysis-requiring AKI) as a clinical endpoint based on the valve replacement approach. Exclusion criteria we applied are (1) observational studies reporting nonpropensity-matched populations and (2) nonpublished studies (abstracts). The following study characteristics were extracted: year of publication, study design, number of patients, clinical characteristics, confounding factors, comparability between groups at baseline, outcomes, and study follow-up. The main outcomes of interest between the two interventions in this study included (1) incidence of AKI at 30 days and (2) incidence of $\mathrm{AKI}$ requiring dialysis at 30 days.

\section{Data Synthesis and Analysis}

The data supporting this meta-analysis are from previously reported studies and datasets, which have been cited. The processed data are reported in the article and in the supplementary files. We performed our meta-analyses using Comprehensive Meta-Analysis version 3.0 (Biostat, https://www.meta-analysis.com). We used the random effects model with the Mantel-Haenszel (MH) method for each clinical endpoint and pooled estimates of odds ratio (OR) with $95 \%$ confidence interval (CI) were calculated. We used $\mathrm{I}^{2}$ index, tau squared, and the Q-test $P$ value to examine heterogeneity among individual study effect sizes. To reduce the risk of bias, we undertook independent pooling of data from RCTs and PSM observational studies. In order to formally assess publication bias we prepared funnel plots and Egger's linear regression test of funnel plot asymmetry (eFigures 1 and 2). All pooled estimates are displayed with a $95 \%$ confidence interval (CI). $P$ values were considered statistically significant at less than 0.05 . We also performed sensitivity analysis to investigate potential sources of inconsistency, including removal of nonrandomized studies. Forest plots were generated to show the relative effect size of TAVR and SAVR for each clinical outcome. Potential sources of heterogeneity were investigated using meta-regression techniques; factors analyzed in the metaregression included age, sex, diabetes, prior stroke, chronic renal insufficiency, vascular disease, and atrial fibrillation (eFigure 3). We followed standard protocol for performing meta-analysis as in our previous publication. The endpoint of interest in this study is renal outcomes which were not described in our previous papers making this study unique. $[5,6]$

\section{Results}

A total of 5,067 potentially relevant citations were identified and screened (Figure 1). After removal of duplicated studies, we retrieved 76 full-text articles for evaluation, of which 26 satisfied the selection criteria. A total of 20 PSM observational studies and 6 RCTs were included in the meta-analysis (Figure 1). All eligible studies were in the English Language. The baseline characteristics of the patients in the included studies are summarized in Table 1 . The 26 studies enrolled a total of 19,954 patients; 10,038 (50.3\%) in the TAVR group and $9,916(49.7 \%)$ in the SAVR group. Sample sizes ranged from 28 to 4732 patients. Mean age was $79.1 \pm 5.8$ and 78.1 \pm 5.9 years in the TAVR and SAVR groups, respectively $(\mathrm{p}=0.53)$. There was no significant difference in the prevalence of key morbidities between the two groups including chronic 


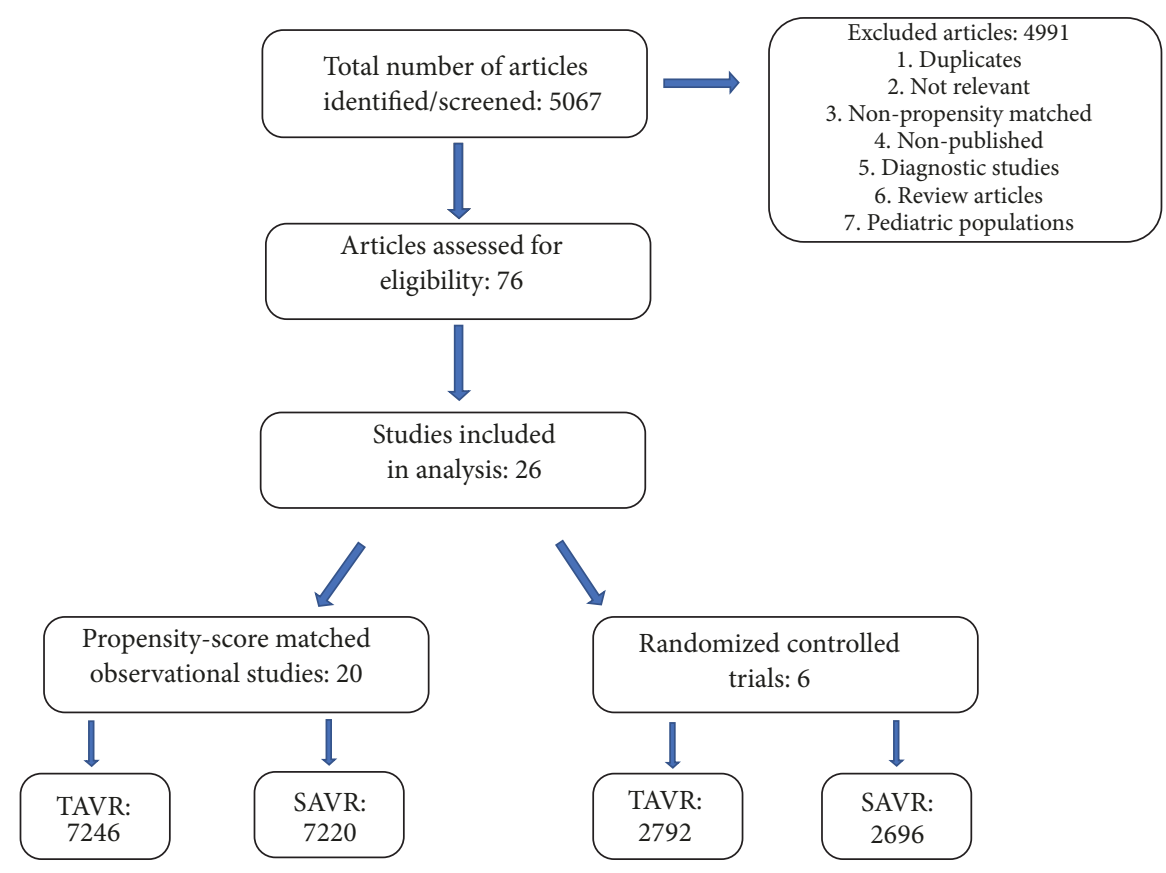

FIGURE 1: Flow chart of the meta-analysis. TAVR: transcatheter aortic valve replacement, SAVR: surgical aortic valve replacement.

renal insufficiency $(25.9 \%$ in the TAVR group vs. $25.3 \%$ in the SAVR group, $\mathrm{p}=0.94$ ) (Table 1). Detailed baseline characteristics of individual studies included in our metaanalysis are illustrated in eTable 1. [7-32]

4.1. Meta-Analysis of RCT and PSM Studies. Eighteen studies (5 RCTs and 13 PSM observational studies; 4,633 TAVR patients; 4,724 SAVR patients) reported the incidence of AKI at 30 days. The pooled estimated incidence of AKI among these studies was 7.1\% after TAVR and $12.1 \%$ after SAVR (OR 0.52; 95\%CI, 0.39-0.68; $\mathrm{p}<0.001)\left(\mathrm{I}^{2}=57 \%\right.$ ) (Figure 2). Seventeen studies (2 RCTs and 15 PSM observational studies; 7,129 TAVR patients; 7,312 SAVR patients) reported the incidence of dialysis-requiring AKI at 30 days, which was similar between patients who underwent TAVR and those who underwent SAVR (2.8\% vs. 4.1\%, OR 0.78; 95\% CI, 0.49$1.25 ; \mathrm{p}=0.31)\left(\mathrm{I}^{2}=70 \%\right)$ (Figure 3$)$. In the meta-regression, age, sex, and the diabetes, prior stroke, chronic renal insufficiency, vascular disease, and atrial fibrillation did not explain the observed heterogeneity between the studies (Supplementary Figures).

4.2. Meta-Analysis of RCT Only. A sensitivity analysis was performed by excluding PSM studies and restricting the meta-analysis to RCTs only. Similar to the original analysis, this meta-analysis showed significantly lower incidence of AKI after TAVR than after SAVR (5 RCTs, 5,418 patients, $2.0 \%$ vs. 5.0\%, OR 0.39; 95\%CI, 0.28-0.53; p<0.001) (Figure 4(a)), but comparable rates of dialysis-requiring AKI (2 studies; 769 patients; $2.9 \%$ vs. $2.6 \%$, OR 1.1; 95\% CI, 0.47-2.58; $\mathrm{p}=0.83$ ) (Figures $4(\mathrm{a})$ and $4(\mathrm{~b})$ ). No heterogeneity among these trials was observed $\left(\mathrm{I}^{2}=0 \%\right)$.
4.3. Meta-Analysis Stratified by Surgical Risk. A secondary analysis was performed to compare the pooled incidence of AKI and dialysis-requiring AKI among patients who are at high surgical risk and those at low-intermediate surgical risk.

(A) Renal outcomes in high-surgical risk patients: seven studies including 2,787 patients reported the incidence of AKI in high-surgical risk patients who underwent TAVR vs. SAVR. In these studies, TAVR was associated with lower pooled incidence of AKI (5.5\% vs. $11 \%$, OR 0.45, 95\%CI 0.25 $0.83, \mathrm{p}=0.01, \mathrm{I}^{2}=68 \%$ ) (Figure $5(\mathrm{a})$ ). Seven studies including 2,407 patients reported the incidence of dialysis-requiring AKI after valve replacement in high-surgical risk patients. In these studies, there was no significant difference in the pooled incidence of dialysis-requiring AKI between TAVR and SAVR (7.4\% vs. $6.2 \%$, OR 0.95, 95\%CI 0.42-2.16, $\mathrm{p}=0.91$, $\left.\mathrm{I}^{2}=78 \%\right)$ (Figure 6(a)).

(B) Renal outcomes in low-intermediate surgical risk patients: ten studies including 6,510 patients reported the incidence of AKI following TAVR vs. SAVR in lowintermediate surgical risk patients that compared with SAVR, TAVR was associated with lower pooled incidence of AKI (7.6\% vs. $12.4 \%$, OR $0.55,95 \%$ CI $0.39-0.77, \mathrm{P}<0.001, \mathrm{I}^{2}=57 \%$ ) (Figure 5(b)). Also, in the ten studies ( $\mathrm{n}=12,034$ patients) that reported the incidence of dialysis-requiring AKI in this cohort of patient, TAVR was associated with significantly lower incidence of dialysis-requiring AKI compared with SAVR $(2.0 \%$ vs. $3.6 \%$, OR $0.57,95 \%$ CI $0.38-0.85, \mathrm{p}=0.005$, $\left.\mathrm{I}^{2}=23 \%\right)$ (Figure 6(b)).

\section{Discussion}

The major findings of the current investigation are as follows. (1) TAVR is associated with lower rates of AKI compared with 
Study name

\begin{tabular}{lcccr}
\hline & TAVR & SAVR & $\begin{array}{c}\text { MH odds } \\
\text { ratio }\end{array}$ & p-Value \\
COREVALVE trial & $23 / 390$ & $54 / 357$ & 0.352 & 0.000 \\
NOTION trial & $1 / 145$ & $9 / 135$ & 0.097 & 0.028 \\
PARTNER 1 trial & $4 / 348$ & $4 / 351$ & 1.009 & 0.990 \\
PARTNER 2A trial & $13 / 1011$ & $31 / 1021$ & 0.416 & 0.009 \\
SURTAVI trial & $15 / 864$ & $35 / 796$ & 0.384 & 0.002 \\
Ailawadi et al & $17 / 340$ & $29 / 340$ & 0.564 & 0.070 \\
Appel et al & $4 / 45$ & $5 / 45$ & 0.780 & 0.726 \\
Bagur et al & $11 / 119$ & $27 / 104$ & 0.290 & 0.001 \\
Fraccaro et al & $105 / 415$ & $184 / 415$ & 0.425 & 0.000 \\
Fusari et al & $4 / 30$ & $5 / 30$ & 0.769 & 0.718 \\
Latib et al & $9 / 111$ & $29 / 111$ & 0.249 & 0.001 \\
Muneretto et al & $24 / 204$ & $41 / 408$ & 1.193 & 0.516 \\
Onorati et al & $1 / 28$ & $6 / 28$ & 0.136 & 0.074 \\
Papadopoulos et al & $1 / 40$ & $2 / 40$ & 0.487 & 0.564 \\
Santarpino et al & $1 / 102$ & $5 / 102$ & 0.192 & 0.135 \\
Schymik et al & $44 / 216$ & $39 / 216$ & 1.161 & 0.542 \\
Thakkar et al & $6 / 30$ & $9 / 30$ & 0.583 & 0.374 \\
Thongprayoon et al & $47 / 195$ & $58 / 195$ & 0.750 & 0.210 \\
& $330 / 4633$ & $572 / 4724$ & 0.517 & 0.000
\end{tabular}

Heterogeneity: $\operatorname{Tau}^{2}=0.162 ; d f=17(P=0.001) ;\left.\right|^{2}=57.335$

Events / Total $\mid$
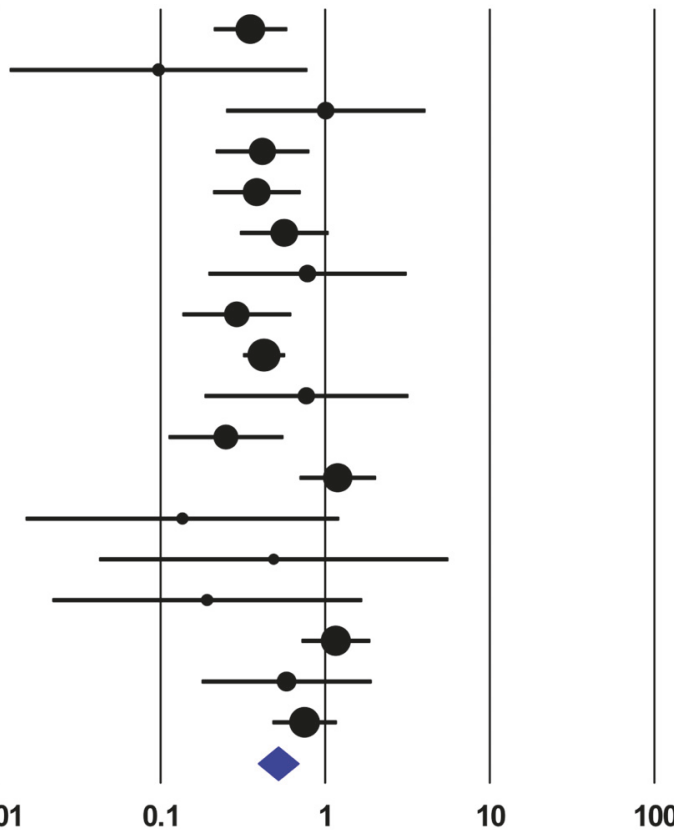

Favors TAVR

Favors SAVR

FIGURE 2: Pooled effect estimates for 30-day acute kidney injury according to the type of aortic valve replacement procedure. TAVR: transcatheter aortic valve replacement, SAVR: surgical aortic valve replacement.

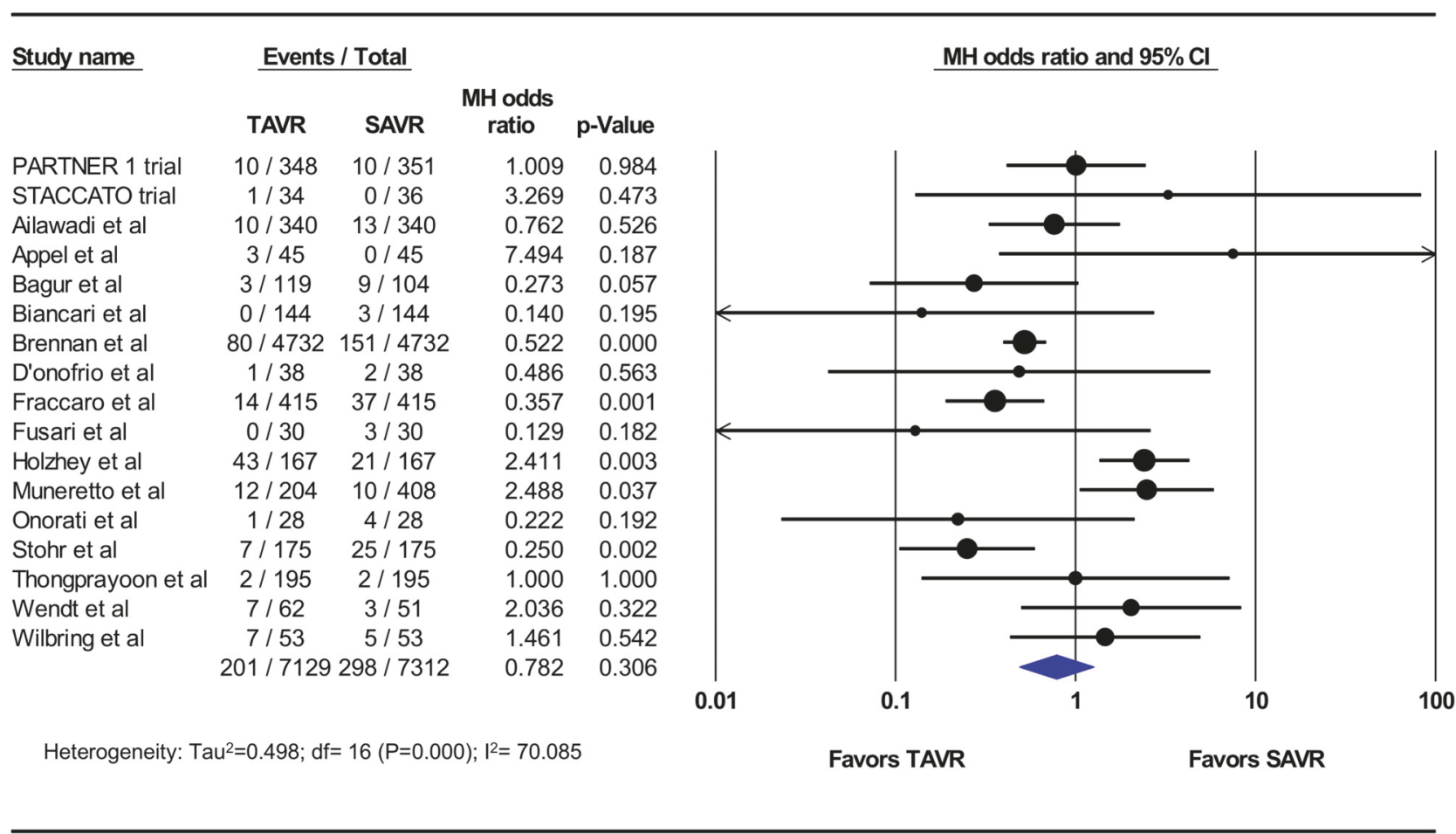

FIGURE 3: Pooled effect estimates for 30-day renal replacement therapy according to the type of aortic valve replacement procedure. TAVR: transcatheter aortic valve replacement, SAVR: surgical aortic valve replacement. 


\begin{tabular}{|c|c|c|c|c|}
\hline \multirow[t]{2}{*}{ Study name } & \multicolumn{2}{|c|}{ Events / Total } & \multirow[b]{2}{*}{$\begin{array}{l}\text { MH odds } \\
\text { ratio }\end{array}$} & \multirow[b]{2}{*}{ p-Value } \\
\hline & TAVR & SAVR & & \\
\hline COREVALVE trial & 23 / 390 & 54 / 357 & 0.352 & 0.000 \\
\hline NOTION trial & $1 / 145$ & $9 / 135$ & 0.097 & 0.028 \\
\hline PARTNER 1 trial & $4 / 348$ & $4 / 351$ & 1.009 & 0.990 \\
\hline PARTNER 2A trial & $13 / 1011$ & $31 / 1021$ & 0.416 & 0.009 \\
\hline \multirow[t]{2}{*}{ SURTAVI trial } & $15 / 864$ & $35 / 796$ & 0.384 & 0.002 \\
\hline & $56 / 2758$ & $133 / 2660$ & 0.385 & 0.000 \\
\hline
\end{tabular}

Heterogeneity: $\operatorname{Tau}^{2}=0.000 ; \mathrm{df}=4(\mathrm{P}=0.447) ; 1^{2}=0.000$

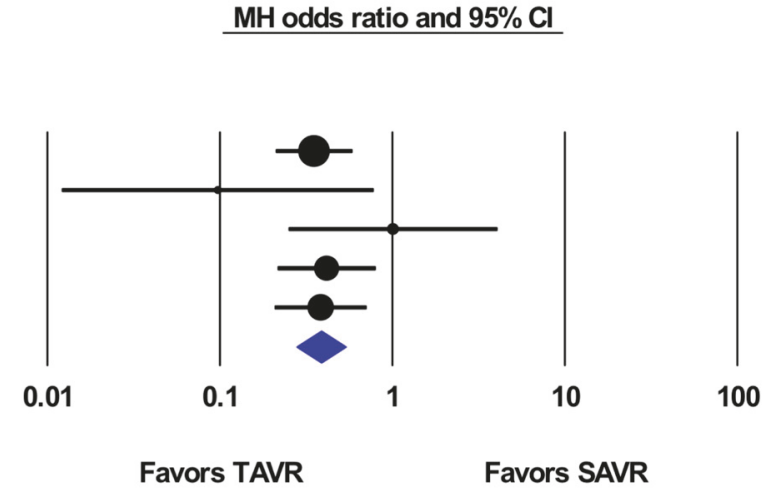

MH odds ratio and $95 \% \mathrm{Cl}$

Favors TAVR

(a)

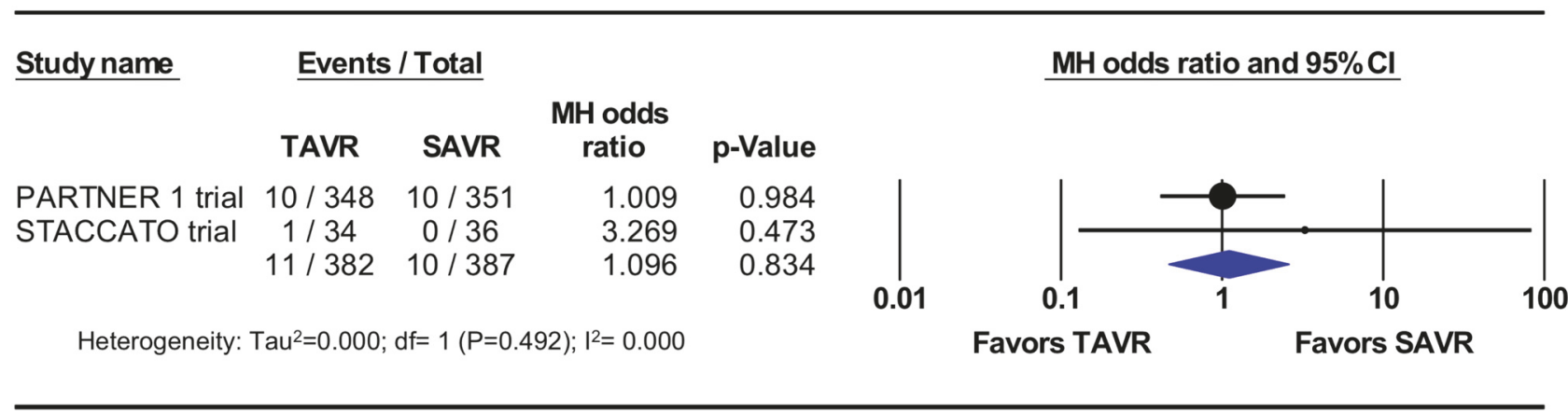

(b)

Figure 4: Pooled effect estimates for 30-day acute kidney injury and renal replacement therapy according to the type of aortic valve replacement procedure in the randomized controlled trials. TAVR: transcatheter aortic valve replacement, SAVR: surgical aortic valve replacement.

SAVR, and this was consistent in the overall analysis, in a sensitivity analysis including RCTs only, and in subanalyses of high-risk and low-intermediate risk patients. (2) The risk of dialysis-requiring AKI appears to be comparable after TAVR vs. SAVR. However, the pooled incidence of dialysisrequiring AKI was significantly lower after TAVR than after SAVR in a subgroup of low-intermediate risk patients.

Patients with severe aortic stenosis are characteristically older and have many comorbidities including a high prevalence of chronic renal insufficiency. Cardiac surgery operations including SAVR are associated with significant risk of AKI and AKI requiring dialysis $[33,34]$. Transcatheter aortic valve replacement was introduced as an effective alternative to surgery in high-prohibitive risk patients but later expanded into young and lower risk patient cohorts. Nonetheless, both the preoperative work-up and the TAVR procedure itself carry a significant risk of AKI due to contrast medium usage, and the high prevalence of atherosclerotic risk factors among patients submitted for TAVR. Whether TAVR is associated with lower risk of AKI and AKI requiring dialysis than SAVR has not been well studied. In the pivotal PARTNER-1 trial, no difference in the rate of AKI was observed between TAVR and SAVR. [7] Subsequent RCTs showed lower rates of AKI after TAVR compared with SAVR. We hence performed a systematic review and a meta-analysis to synthesize the best available evidence on renal outcomes following TAVR and SAVR.

Our meta-analysis showed that TAVR is associated with about $50 \%$ reduction in the incidence of AKI compared with SAVR, but a similar rate of dialysis-requiring AKI between the two modalities overall. These findings have important prognostic implications and deserve more scrutiny for several reasons. (1) There is ample evidence that even AKI not requiring dialysis is associated with substantial negative impact on long-term outcomes [35-41]. (2) The risk of AKI and dialysis-requiring AKI may be more modifiable in patients undergoing TAVR. The advances in 3D echocardiography and the refinements in TAVR techniques have allowed the introduction of the 'Reno-protective TAVR' concept [4246]. This concept along with the wide adoption of moderate sedation in TAVR procedures has the potential to further reduce postprocedural renal insufficiency although this has not yet been studied in a prospective fashion [47]. In contrast, the risk of AKI after SAVR may be more related to the patient risk profile than to modifiable procedural factors as surgical techniques in SAVR have not differed significantly 


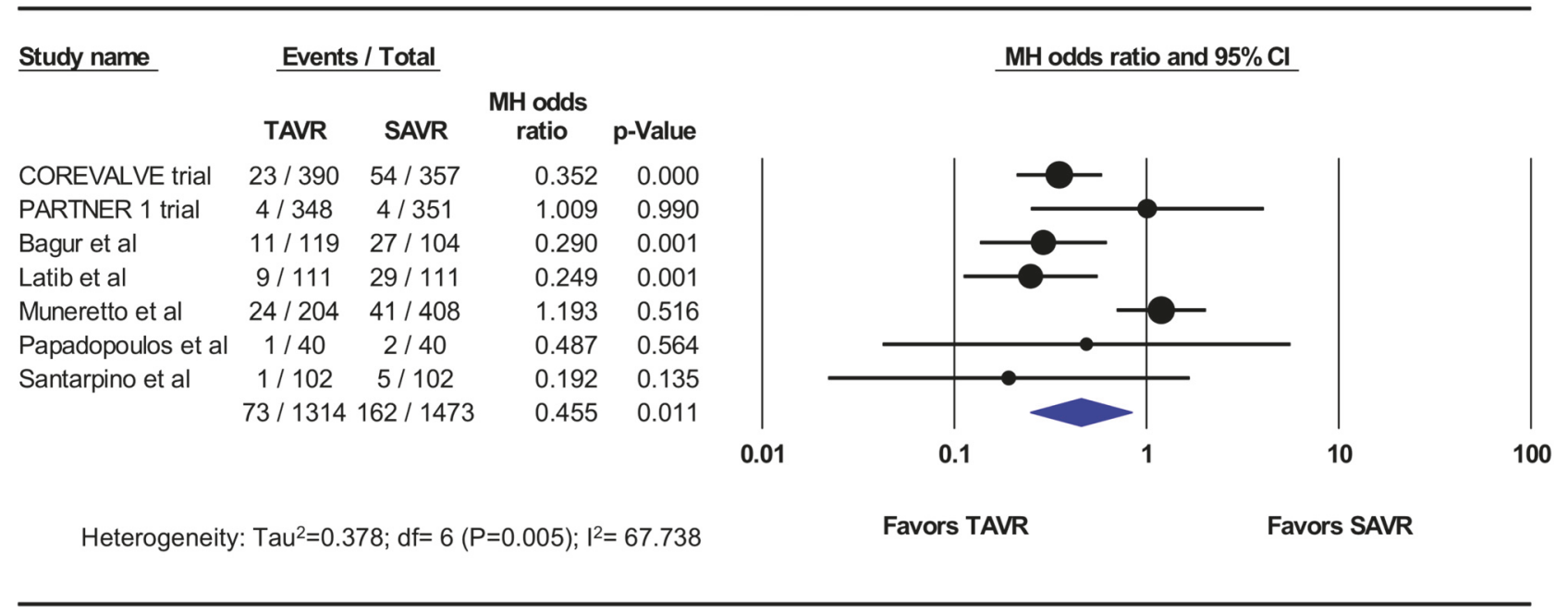

(a)

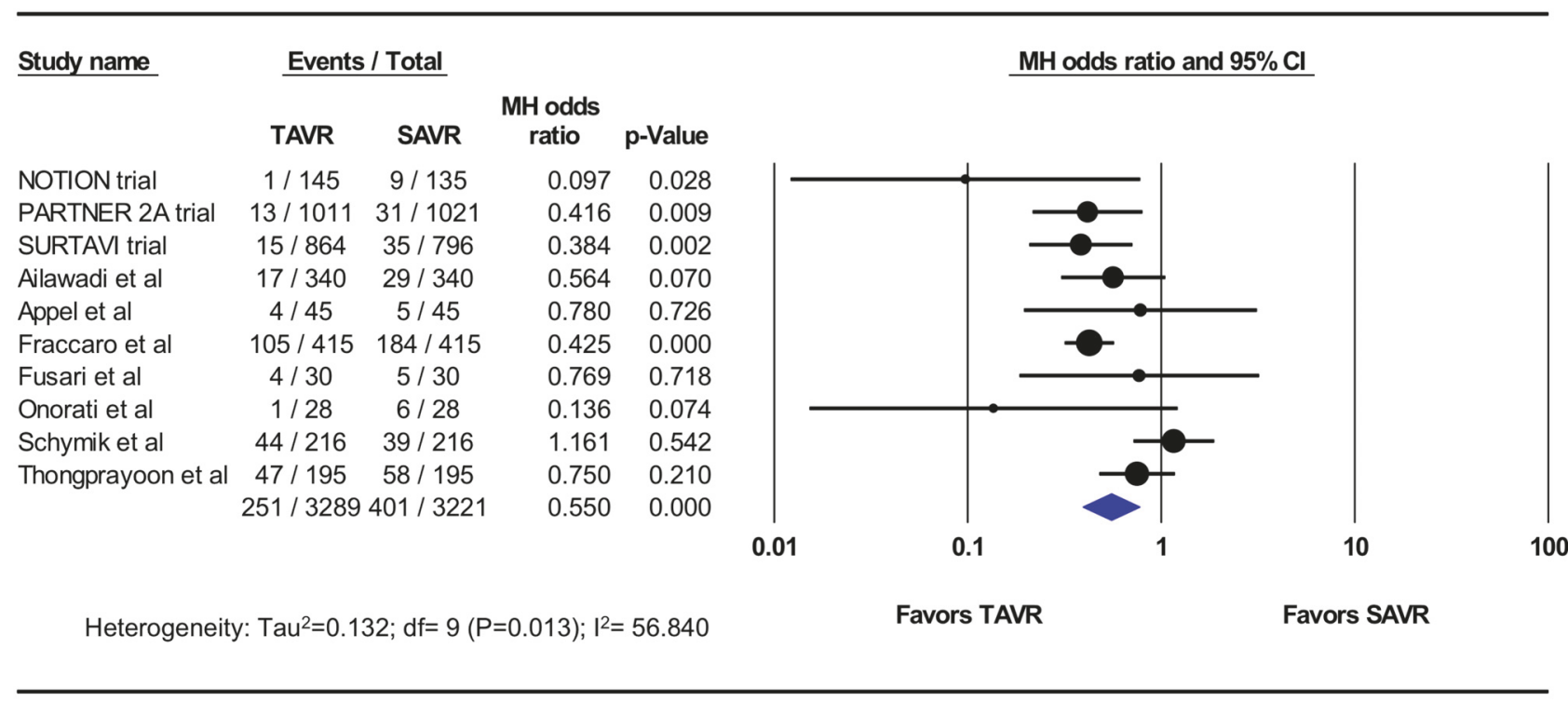

(b)

FIGURE 5: Pooled effect estimates for 30-day acute kidney injury according to the type of aortic valve replacement procedure in the randomized controlled trials. TAVR: transcatheter aortic valve replacement, SAVR: surgical aortic valve replacement.

in the last decade. (3) In our subanalysis of patients who are at low-intermediate surgical risk, TAVR was associated with a significant reduction not only in AKI but also in AKI requiring dialysis. In light of the continuous expansion of TAVR to lower risk populations, the impact of the TAVR on improving renal outcomes in these patients warrants more investigation [48].

\section{Limitations}

Our study has several limitations: (1) there are only few RCTs comparing TAVR with SAVR. Hence, we included observational studies in our meta-analysis. However, we limited our inclusion of observational studies to those with propensity score matched comparisons. While this can introduce heterogeneity into our analysis, our sensitivity analysis including RCTs only yielded similar results to the overall meta-analysis. (2) The definition of AKI varies among the studies, but those definitions were maintained the same in the same study for comparison between TAVR and SAVR, and hence the results are comparable.

\section{Conclusions}

TAVR procedure has significantly lower rates of AKI compared to SAVR but similar rates of AKI requiring renal replacement therapy. AKI has short and long-term effects on 


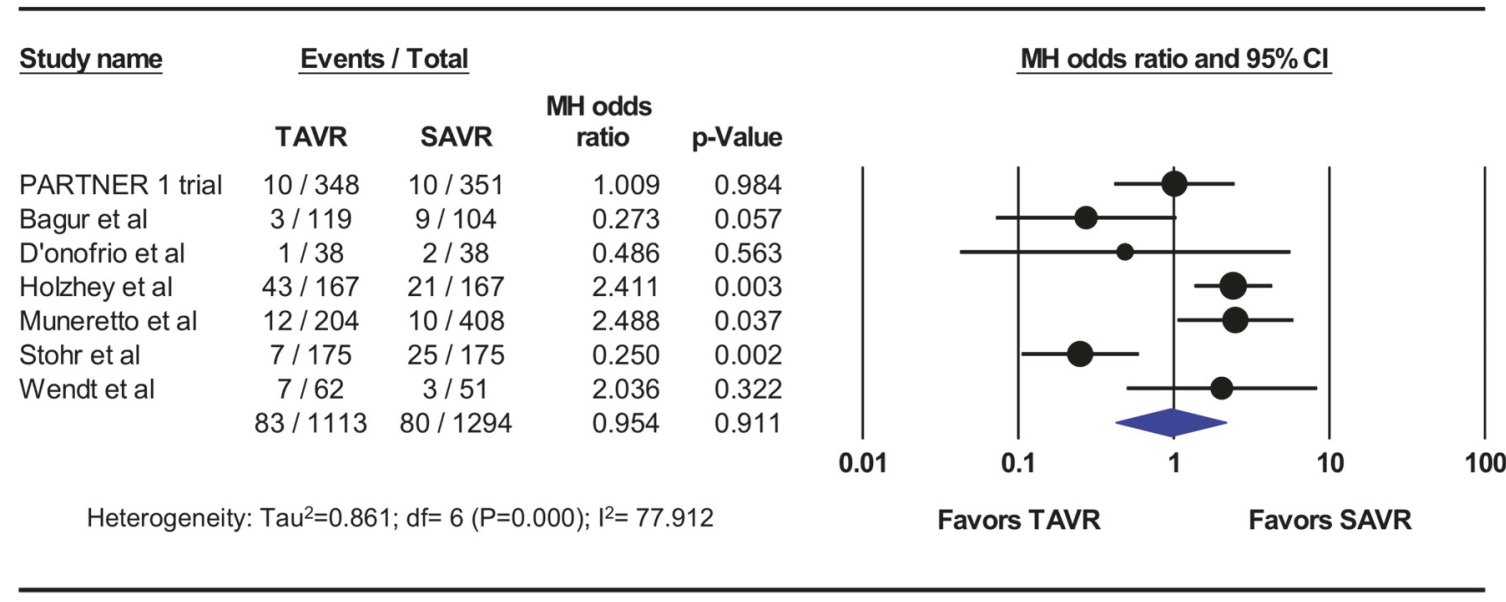

(a)

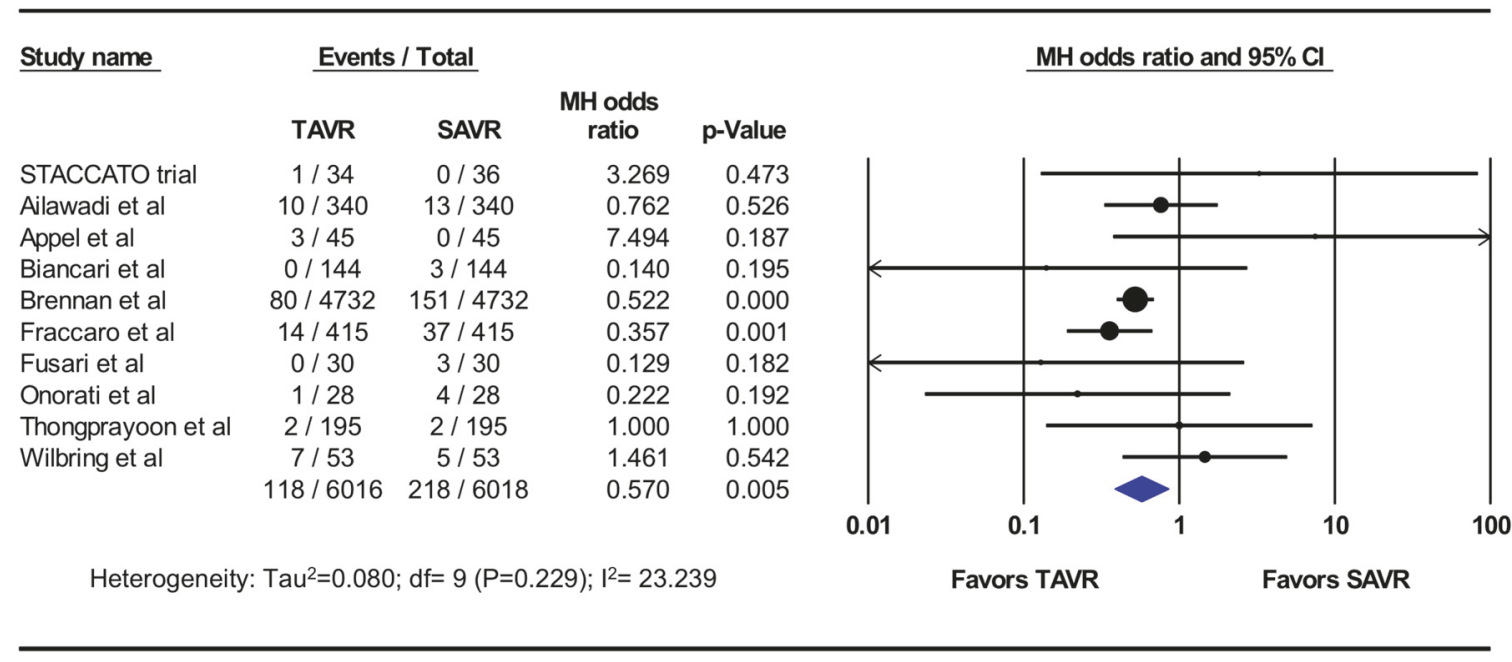

(b)

Figure 6: Pooled effect estimates for 30-day renal replacement therapy according to the type of aortic valve replacement procedure and surgical risk. TAVR: transcatheter aortic valve replacement, SAVR: surgical aortic valve replacement.

outcomes and survival; hence every effort should be made to reduce the incidence of $\mathrm{AKI}$.

\section{Data Availability}

The data used to support the findings of this study are available from the corresponding author upon request.

\section{Disclosure}

All authors listed meet the authorship criteria according to the latest guidelines of the International Committee of Medical Journal Editors, and all authors are in agreement with the manuscript. The article is processing charges to be covered in the institutional membership at West Virginia University.

\section{Conflicts of Interest}

The authors declare that they have no conflicts of interest.

\section{Authors' Contributions}

Dr. Shah and Dr. Chaker contributed equally to this manuscript.

\section{Supplementary Materials}

eFigure 1: Funnel plot of the meta-analysis of the published studies reporting 30-day acute kidney injury in patients undergoing TAVR versus SAVR. TAVR: transcatheter aortic valve replacement; SAVR: surgical aortic valve replacement. eFigure 2: Funnel plot of the meta-analysis of the published studies reporting 30-day renal replacement therapy in patients undergoing TAVR versus SAVR. TAVR: transcatheter aortic valve replacement; SAVR: surgical aortic valve replacement. eFigure 3: Meta regression for age, gender, previous stroke, peripheral arterial disease, diabetes, chronic kidney disease, atrial fibrillation, and acute kidney injury and renal replacement therapy in patients 
undergoing TAVR versus SAVR. TAVR: transcatheter aortic valve replacement; SAVR: surgical aortic valve replacement. (Supplementary Materials)

\section{References}

[1] D. R. Holmes Jr., M. J. MacK, S. Kaul et al., "2012 ACCF/AATS/SCAI/STS expert consensus document on transcatheter aortic valve replacement: developed in collaboration with the american heart association, american society of echocardiography, european association for cardiothoracic surgery, heart failure society of america, mended hearts, society of cardiovascular anesthesiologists," The Annals of Thoracic Surgery, vol. 93, no. 4, pp. 1340-1395, 2012.

[2] R. A. Nishimura, C. M. Otto, R. O. Bonow et al., "AHA/ACC guideline for the management of patients with valvular heart disease: a report of the American College of Cardiology/American Heart Association Task Force on Practice Guidelines," Journal of the American College of Cardiology, vol. 63, pp. e57-185, 2014.

[3] N. Kumar and N. Garg, "Acute kidney injury after aortic valve replacement in a nationally representative cohort in the USA," Nephrology Dialysis Transplantation, 2018.

[4] D. Moher, A. Liberati, J. Tetzlaff, and D. G. Altman, "Preferred reporting items for systematic reviews and meta-analyses: the PRISMA statement," International Journal of Surgery, vol. 8, no. 5, pp. 336-341, 2010.

[5] K. Shah, Z. Chaker, T. Busu et al., "Meta-analysis comparing the frequency of stroke after transcatheter versus surgical aortic valve replacement," American Journal of Cardiology, vol. 122, no. 7, pp. 1215-1221, 2018.

[6] T. Busu, F. Alqahtani, V. Badhwar, C. C. Cook, C. S. Rihal, and M. Alkhouli, "Meta-analysis comparing transcatheter and surgical treatments of paravalvular leaks," American Journal of Cardiology, vol. 122, no. 2, pp. 302-309, 2018.

[7] C. R. Smith, M. B. Leon, M. J. Mack et al., "Transcatheter versus surgical aortic-valve replacement in high-risk patients," The New England Journal of Medicine, vol. 364, pp. 2187-2198, 2011.

[8] H. H. M. Nielsen, K. E. Klaaborg, H. Nissen et al., "A prospective, randomised trial of transapical transcatheter aortic valve implantation vs. surgical aortic valve replacement in operable elderly patients with aortic stenosis: The STACCATO trial," EuroIntervention, vol. 8, no. 3, pp. 383-389, 2012.

[9] D. H. Adams, J. J. Popma, M. J. Reardon et al., "Transcatheter aortic-valve replacement with a self-expanding prosthesis," The New England Journal of Medicine, vol. 370, pp. 1790-1798, 2014.

[10] H. G. Thyregod, D. A. Steinbruchel, and N. Ihlemann, “Transcatheter versus surgical aortic valve replacement in patients with severe aortic valve stenosis: 1-year results from the allcomers notion randomized clinical trial," Journal of the American College of Cardiology, vol. 65, pp. 2184-2194, 2015.

[11] M. B. Leon, C. R. Smith, and M. J. Mack, "Transcatheter or surgical Aortic-valve replacement in intermediate-risk patients," The New England Journal of Medicine, vol. 374, pp. 1609-1620, 2016.

[12] M. J. Reardon, N. M. Van Mieghem, J. J. Popma et al., "Surgical or transcatheter aortic-valve replacement in intermediate-risk patients," The New England Journal of Medicine, pp. 1321-1331, 2017.

[13] R. Bagur, J. G. Webb, F. Nietlispach et al., "Acute kidney injury following transcatheter aortic valve implantation: Predictive factors, prognostic value, and comparison with surgical aortic valve replacement," European Heart Journal, vol. 31, no. 7, pp. 865-874, 2010.

[14] M. Fusari, V. Bona, M. Muratori et al., "Transcatheter vs. surgical aortic valve replacement: A retrospective analysis assessing clinical effectiveness and safety," Journal of Cardiovascular Medicine, vol. 13, no. 4, pp. 229-241, 2012.

[15] R. Stöhr, G. Dohmen, R. Herpertz et al., "Thirty-day outcome after transcatheter aortic valve implantation compared with surgical valve replacement in patients with high-risk aortic stenosis: A matched comparison," Coronary Artery Disease, vol. 22, no. 8, pp. 595-600, 2011.

[16] D. M. Holzhey, W. Shi, A. Rastan, M. A. Borger, M. Hänsig, and F. W. Mohr, "Transapical versus conventional aortic valve replacement - A propensity-matched comparison," Heart Surgery Forum, vol. 15, no. 1, p. -E8, 2012.

[17] C.-F. Appel, H. Hultkvist, E. Nylander et al., "Transcatheter versus surgical treatment for aortic stenosis: Patient selection and early outcome*," Scandinavian Cardiovascular Journal, vol. 46, no. 5, pp. 301-307, 2012.

[18] A. Latib, F. Maisano, L. Bertoldi et al., "Transcatheter vs surgical aortic valve replacement in intermediate- surgical-risk patients with aortic stenosis: A propensity score-matched case-control study," American Heart Journal, vol. 164, no. 6, pp. 910-917, 2012.

[19] A. D’Onofrio, A. Messina, R. Lorusso et al., "Sutureless aortic valve replacement as an alternative treatment for patients belonging to the "gray zone" between transcatheter aortic valve implantation and conventional surgery: A propensitymatched, multicenter analysis," The Journal of Thoracic and Cardiovascular Surgery, vol. 144, no. 5, pp. 1010-1018, 2012.

[20] M. Wilbring, S.-M. Tugtekin, K. Alexiou, G. Simonis, K. Matschke, and U. Kappert, "Transapical transcatheter aortic valve implantation vs conventional aortic valve replacement in high-risk patients with previous cardiac surgery: A propensityscore analysis," European Journal of Cardio-Thoracic Surgery, vol. 44, no. 1, Article ID ezs680, pp. 42-47, 2013.

[21] N. Papadopoulos, N. Schiller, S. Fichtlscherer et al., "Propensity matched analysis of longterm outcomes following transcatheter based aortic valve implantation versus classic aortic valve replacement in patients with previous cardiac surgery," Journal of Cardiothoracic Surgery, vol. 9, no. 1, p. 99, 2014.

[22] G. Santarpino, S. Pfeiffer, J. Jessl et al., "Clinical outcome and cost analysis of sutureless versus transcatheter aortic valve implantation with propensity score matching analysis," American Journal of Cardiology, vol. 116, no. 11, pp. 1737-1743, 2015.

[23] G. Schymik, M. Heimeshoff, P. Bramlage et al., "A comparison of transcatheter aortic valve implantation and surgical aortic valve replacement in 1,141 patients with severe symptomatic aortic stenosis and less than high risk," Catheterization and Cardiovascular Interventions, vol. 86, no. 4, pp. 738-744, 2015.

[24] C. Muneretto, O. Alfieri, B. M. Cesana et al., "A comparison of conventional surgery, transcatheter aortic valve replacement, and sutureless valves in "real-world" patients with aortic stenosis and intermediate- to high-risk profile," The Journal of Thoracic and Cardiovascular Surgery, vol. 150, no. 6, pp. 15701579, 2015.

[25] D. Wendt, F. Al-Rashid, P. Kahlert et al., "Conventional aortic valve replacement or transcatheter aortic valve implantation in patients with previous cardiac surgery," Journal of Cardiology, vol. 66, no. 4, pp. 292-297, 2015.

[26] B. Thakkar, A. Patel, B. Mohamad et al., "Transcatheter aortic valve replacement versus surgical aortic valve replacement in 
patients with cirrhosis," Catheterization and Cardiovascular Interventions, vol. 87, no. 5, pp. 955-962, 2016.

[27] C. Thongprayoon, W. Cheungpasitporn, N. Srivali et al., "AKI after transcatheter or surgical aortic valve replacement," Journal of the American Society of Nephrology, vol. 27, no. 6, pp. 18541860, 2016.

[28] F. Biancari, M. Barbanti, G. Santarpino et al., "Immediate outcome after sutureless versus transcatheter aortic valve replacement," Heart and Vessels, vol. 31, no. 3, pp. 427-433, 2016.

[29] F. Onorati, A. DOnofrio, F. Biancari et al., "Results of surgical aortic valve replacement and transapical transcatheter aortic valve replacement in patients with previous coronary artery bypass grafting," Interact Cardiovasc Thorac Surg, vol. 22, pp. 806-812, 2016.

[30] C. Fraccaro, G. Tarantini, S. Rosato et al., "Early and Midterm Outcome of Propensity-Matched Intermediate-Risk Patients Aged $\geq 80$ Years with Aortic Stenosis Undergoing Surgical or Transcatheter Aortic Valve Replacement (from the Italian Multicenter OBSERVANT Study)," American Journal of Cardiology, vol. 117, no. 9, pp. 1494-1501, 2016.

[31] G. Ailawadi, D. J. LaPar, A. M. Speir et al., "Contemporary costs associated with transcatheter aortic valve replacement," The Annals of Thoracic Surgery, vol. 101, no. 1, pp. 154-161, 2016.

[32] J. M. Brennan, L. Thomas, and DJ. Cohen, "Transcatheter versus surgical aortic valve replacement: propensity-matched comparison," Journal of the American College of Cardiology, vol. 70, pp. 439-450, 2017.

[33] W. Reents, M. Hilker, J. Börgermann et al., "Acute kidney injury after on-pump or off-pump coronary artery bypass grafting in elderly patients," The Annals of Thoracic Surgery, vol. 98, no. 1, pp. 9-15, 2014.

[34] M. Vives, D. Wijeysundera, N. Marczin, P. Monedero, and V. Rao, "Cardiac surgery-associated acute kidney injury," Interactive CardioVascular and Thoracic Surgery, vol. 18, no. 5, pp. 637645, 2014.

[35] Y.-B. Liao, X.-X. Deng, Y. Meng et al., "Predictors and outcome of acute kidney injury after transcatheter aortic valve implantation: A systematic review and meta-analysis," EuroIntervention, vol. 12, no. 17, pp. 2067-2074, 2017.

[36] K. Chatani, M. Abdel-Wahab, N. Wübken-Kleinfeld et al., "Acute kidney injury after transcatheter aortic valve implantation: Impact of contrast agents, predictive factors, and prognostic importance in 203 patients with long-term follow-up," Journal of Cardiology, vol. 66, no. 6, pp. 514-519, 2015.

[37] M. Konigstein, E. Ben-Assa, S. Banai et al., "Periprocedural bleeding, acute kidney injury, and long-term mortality after transcatheter aortic valve implantation," Canadian Journal of Cardiology, vol. 31, no. 1, pp. 56-62, 2015.

[38] M. Ma, W. D. Gao, Y. F. Gu, Y. S. Wang, Y. Zhu, and Y. He, "Clinical effects of acute kidney injury after transcatheter aortic valve implantation: a systematic review and meta-analysis," Internal and Emergency Medicine, 2018.

[39] O. Kliuk-Ben Bassat, A. Finkelstein, and S. Bazan, "Acute kidney injury after transcatheter aortic valve implantation and mortality risk-long-term follow-up," Nephrology Dialysis Transplantation, 2018.

[40] A. C. B. Nunes Filho, M. Katz, C. M. Campos et al., "Impact of acute kidney injury on short- and long-term outcomes after transcatheter aortic valve implantation," Revista Española de Cardiología, 2018.
[41] A. J. Muñoz-García, E. Muñoz-García, M. F. Jiménez-Navarro et al., "Clinical impact of acute kidney injury on short- and longterm outcomes after transcatheter aortic valve implantation with the CoreValve prosthesis," Journal of Cardiology, vol. 66, no. 1, pp. 46-49, 2015.

[42] R. Higuchi, T. Tobaru, K. Hagiya et al., "Renoprotective transcatheter aortic valve implantation without contrast media," International Heart Journal, vol. 59, no. 6, pp. 1469-1472, 2018.

[43] M. S. van Mourik, F. van Kesteren, R. N. Planken et al., "Short versus conventional hydration for prevention of kidney injury during pre-TAVI computed tomography angiography," Netherlands Heart Journal, vol. 26, no. 9, pp. 425-432, 2018.

[44] A. Elkaryoni, N. C. Nanda, P. Baweja et al., "Three-dimensional transesophageal echocardiography is an attractive alternative to cardiac multi-detector computed tomography for aortic annular sizing: Systematic review and meta-analysis," Journal of Echocardiography, vol. 35, no. 10, pp. 1626-1634, 2018.

[45] M. Renker, A. Varga-Szemes, U. J. Schoepf et al., "A noncontrast self-navigated 3-dimensional MR technique for aortic root and vascular access route assessment in the context of transcatheter aortic valve replacement: proof of concept," European Radiology, vol. 26, no. 4, pp. 951-958, 2016.

[46] A. Pershad, G. Fraij, S. V. Girotra, H. K. Fang, and G. Gellert, "TEE-guided transcatheter aortic valve implantation with "Zero Contrast" - A viable alternative for patients with chronic kidney disease," The Journal of Invasive Cardiology, vol. 27, no. 2, pp. E25-E26, 2015.

[47] M. C. Hyman, S. Vemulapalli, W. Y. Szeto et al., "Conscious Sedation Versus General Anesthesia for Transcatheter Aortic Valve Replacement:Insights from the National Cardiovascular Data Registry Society of Thoracic Surgeons/American College of Cardiology Transcatheter Valve Therapy Registry," Circulation, vol. 136, no. 22, pp. 2132-2140, 2017.

[48] D. R. Holmes Jr., R. A. Nishimura, F. L. Grover et al., "Annual Outcomes With Transcatheter Valve Therapy: From the STS/ACC TVT Registry," The Annals of Thoracic Surgery, vol. 101, pp. 789-800, 2016. 


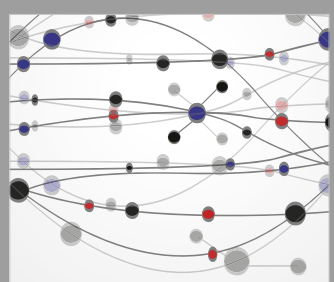

The Scientific World Journal
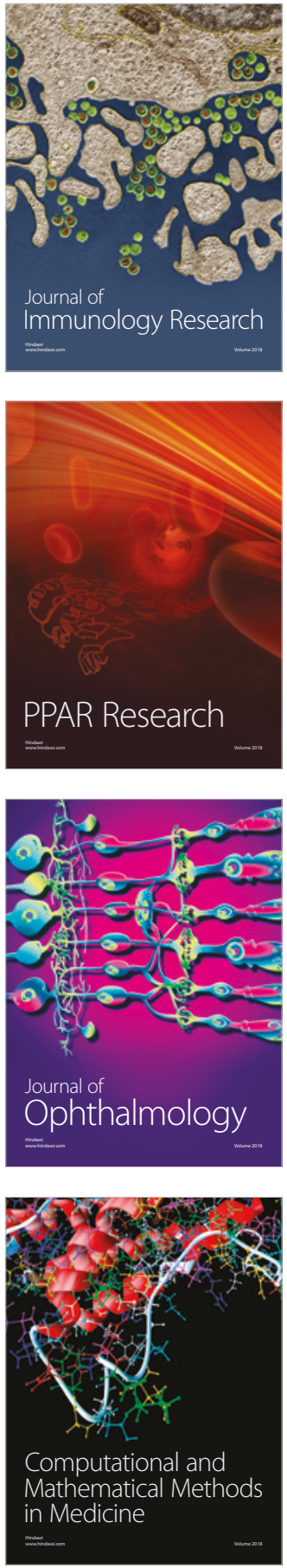

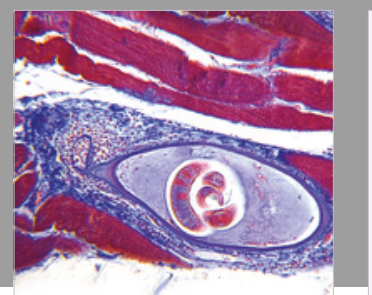

Gastroenterology Research and Practice

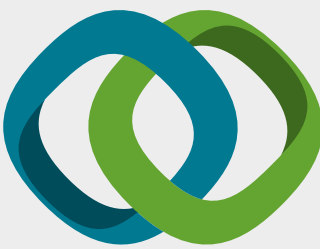

\section{Hindawi}

Submit your manuscripts at

www.hindawi.com
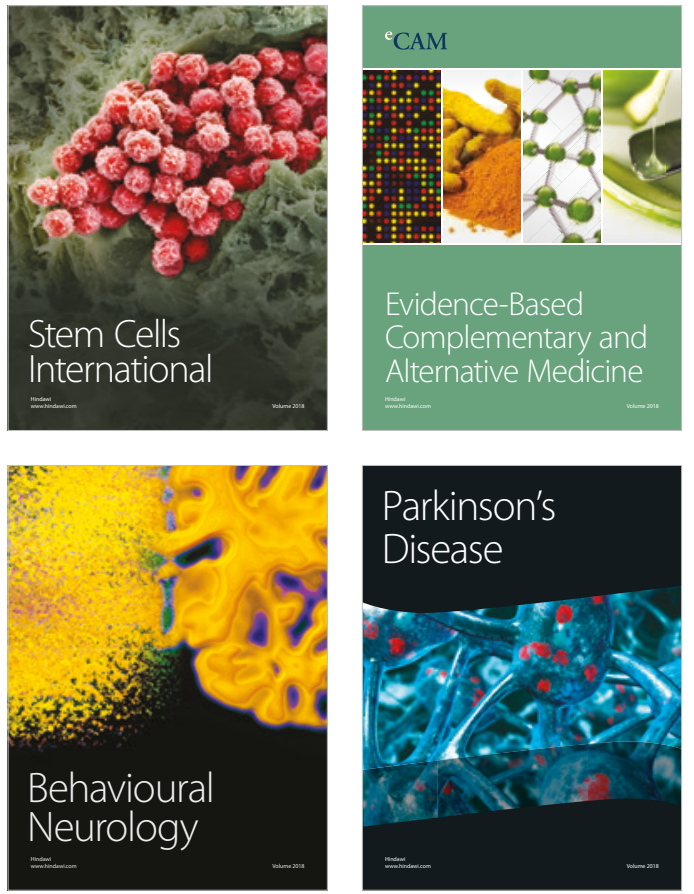

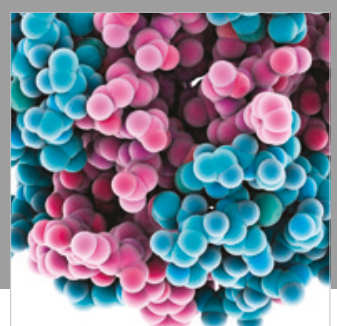

ournal of

Diabetes Research

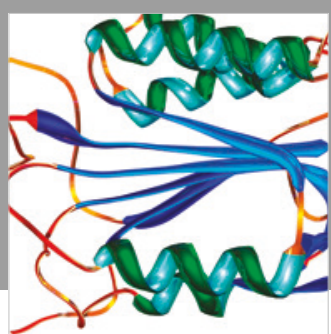

Disease Markers
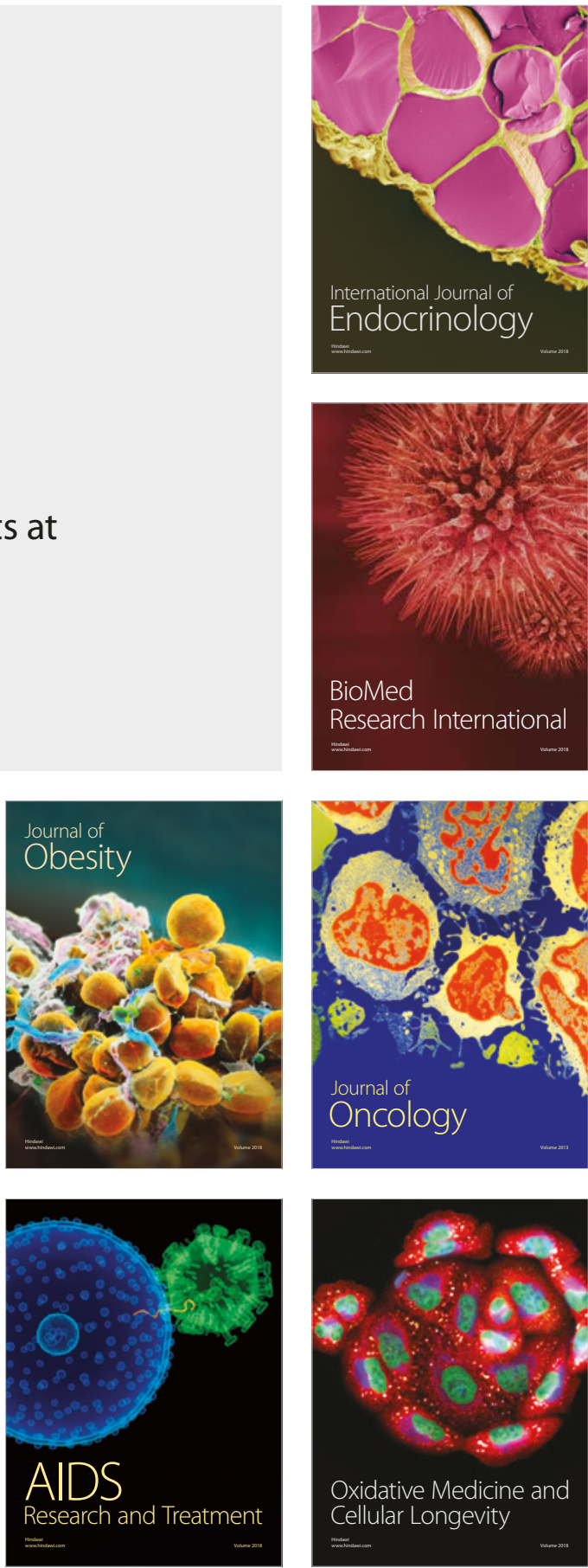